\title{
Electro-Optical Properties of a Transmissive Diffuser based on Electrically-Tuneable Polymer-Dispersed Liquid Crystal Film
}

\author{
Hui LI ${ }^{1,2,4}$ *, Feifan YANG ${ }^{1,2}$, Jing PENG ${ }^{1,2}$, Fan PAN ${ }^{3}$, Yuntao WU ${ }^{1,2}$, \\ Yanduo ZHANG ${ }^{1,2}$, Xiaolin XIE $^{4}$ \\ ${ }^{1}$ School of Computer Science and Engineering, Wuhan Institute of Technology, Wuhan 430205, P.R. China \\ ${ }^{2}$ Hubei Key Laboratory of Intelligent Robot, Wuhan 430205, P.R. China \\ ${ }^{3}$ College of Post and Telecommunication, Wuhan Institute of Technology, Wuhan 430073, P. R. China \\ ${ }^{4}$ School of Chemistry and Chemical Engineering, Huazhong University of Science and Technology, Wuhan 430073, \\ P.R. China
}

crossref http://dx.doi.org/10.5755/j02.ms.23520

\section{Received 04 June 2019; accepted 23 July 2019}

\begin{abstract}
A transmissive diffuser that is based on polymer-dispersed liquid crystal (PDLC) film has been developed. Liquid crystal E7 and polymer NOA65, at a ratio of 55:45 by weight, were utilized to fabricate the diffuser. With the use of phase separation, diffuser of $7.5 \mu \mathrm{m}$ PDLC film thickness can be formed after 20 minutes UV irradiation with an ultraviolet lamp (36E @ 365nm). Test results have shown that the proposed diffuser has good classic electro-optical and transmissive imaging features under an external tuneable electric field. The development has a great application potential in augmented reality or mixed reality-type applications.

Keywords: transmissive diffuser, polymer dispersed liquid crystal, phase separation.
\end{abstract}

\section{INTRODUCTION}

During the past decade, display technology has developed very rapidly [1]. In particular, 3D displays have become the future display trend $[2,3]$. There are so many different kinds of materials and devices with the development of new technologies. In relation to display applications, there is a strong demand for diffusers in some fields, such as for augmented reality (AR) and mixed reality (MR) products $[4,5]$. In a classic AR system, there is a live direct or indirect view of a physical, real-world environment whose informations are augmented (or supplemented) by computer-generated sensory input such as sound, video, graphics or GPS data $[4,5]$. This approach is related to a more general concept called MR, in which a view of reality is modified (possibly even diminished rather than augmented) by a computer. The diffuser is a very important optical element to facilitate fusion between the virtual world and the real world in a classic AR system. In general, polyethylene terephthalate (PET), polycarbonate (PC), and polymethyl methacrylate (PMMA) films are utilized to fabricate the optical diffuser [6-8]. These materials can lead to several problems, such as that they do not have a high transmittance of polarized light and this consequently produces a poor display effect $[9,10]$. In order to resolve the above-mentioned problems, many different methods have been proposed [11-13]. However, there is still an urgent need to develop low-cost and efficient diffuser technology.

Polymer-dispersed liquid-crystal devices (PDLCs) are an excellent choice of photoelectric material for fabricating different kinds of tuneable devices, including optical

\footnotetext{
${ }^{*}$ Corresponding author. Tel.: +86-27-87992007; fax: +86-27-87992007.

E-mail address: lihui00317@163.com(H. Li)
}

diffusers $[14,15]$. For the LC dispersion system, when the LC content is high, the LC is a continuous phase. When the LC content is low, the LC is dispersed in a continuous polymer matrix and the LC droplets are micron size. This is well-known as PDLC. At the present time, the main preparation methods of PDLC are polymerization induced phase separation (PIPS), thermally induced phase separation (TIPS), solvent induced phase separation (SIPS), the encapsulation emulsification method (EM), and templated cavities (TC) $[14,15]$. PDLC has good application prospects in photoelectric display devices, optical switches, optical attenuators, holographic gratings, selective transmission films, etc. $[14,15]$. As a photoelectric display material, PDLC works mainly between the scattering state and the transparent state. Its response rate is 1 to 2 orders of magnitude faster than that of TN-type LCD. Additionally, the gray level is easy to adjust and control, which can greatly improve image quality. Most importantly, PDLC devices do not require polarizers. They can exhibit good brightness and have a large viewing angle, and it is not necessary to align them along a substrate surface during the fabrication process. PDLC is a solid-state film and it can effectively prevent the leakage of the LC. It is easy also to control the thickness of the film and the material is conducive to the production of large-area, ultra-thin and flexible devices. However, PDLC materials have some defects, such as a high driving voltage, slow dynamic response, and hysteresis of transmittance under voltage driving. The question of how to reduce the driving voltage substantially while maintaining high contrast always is a hot issue in current research $[14,15]$. In order to solve the abovementioned problems of conventional optical diffusers, a diffuser based on PDLC film was proposed. LC and monomers were utilized to fabricate the PDLC film and 
some interesting test results for the proposed diffuser were obtained.

In this paper, firstly, the procedure for fabricating the transmissive diffuser based on PDLC film is given in Section 2. In Section 3, the related theory of phase separation and the principle of operation of the PDLC film is described. Section 4 presents some interesting measurements about the new diffuser. Finally, some conclusions are given about the optoelectronic characteristics of the diffuser.

\section{FABRICATION}

The most important step to acquire a transmissive diffuser is to fabricate the PDLC film. There are many methods to fabricate such films. Generally, encapsulation and phase separation methods are proposed ${ }^{[14-17]}$. In the present study, PIPS method was adopted, and LC and monomers were utilized to fabricate the PDLC film with consideration of cost and performance.

The LC E7, from Merck Co., as shown in Fig. 1, was chosen for the present investigation. It is a well-known positive dielectric nematic mixture that has been widely used in the fabrication of several LC devices. Its main features are a rotational viscosity of $232.6 \mathrm{mPa}$.s at $20{ }^{\circ} \mathrm{C}$, an optical birefringence of 0.216 at $20{ }^{\circ} \mathrm{C}$ and $589.3 \mathrm{~nm}$ and a dielectric anisotropy of 14.1 at $20^{\circ} \mathrm{C}$ and $1 \mathrm{KHz}$. The clear point of the material is $58^{\circ} \mathrm{C}$. For E7, the refractive index of ordinary light is $n_{0}=1.521$, and the refractive index of extrordinary light is $n_{\mathrm{e}}=1.746$.

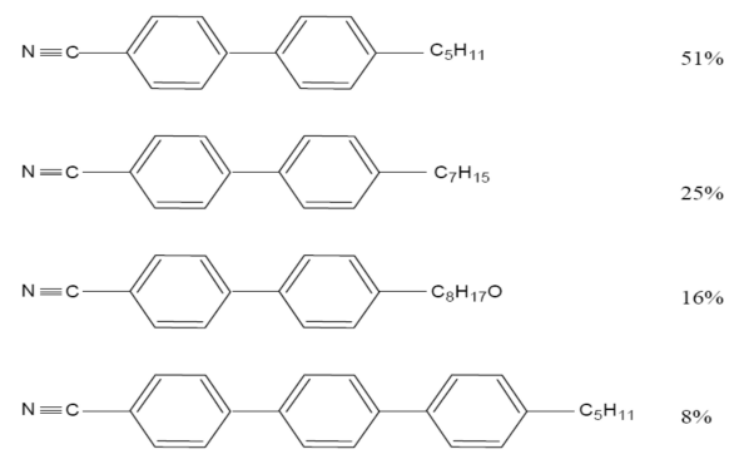

Fig. 1. The structural formula of four components of the commercial LC, E7

The PDLC film was produced by using UV light. During the procedure, the LC, E7, and the polymer, NOA65, from Norland Co., were utilized. The reason for this choice was that the refractive index $n_{\mathrm{p}}$ of the polymer is very close to the refractive index $n_{\mathrm{o}}$ of the LC. After UV-light exposure, the refractive index of NOA65 is $n_{p}=1.524$. For this sample, the mass ratio of 55:45 of the two materials was chosen. The main fabrication process for producing the PDLC cell was as follows: firstly, with the use of an electric balance, the LC and the monomer at a mass ratio of $55 \mathrm{wt} . \%$ : $45 \mathrm{wt} . \%$ were mixed in a glass bottle. An ultrasonic shaker was utilized to agitate the mixture for approximately 4 hours. To ensure uniform mixing, the process of ultrasonic vibration was done four times. Next, an empty cell, composed of two Indium Tin Oxide (ITO) substrates and spacers was prepared. The thickness of the PDLC film was $7.5 \mu \mathrm{m}$. The mixture of
LC-polymer was injected into the empty cell by capillary effect. Finally, AB glue was utilized to seal the LC cell. After a 20-minutes exposure to UV irradiation from an ultraviolet lamp (36W @ 365nm), the sealed cell with a PDLC film was obtained.

\section{RELATED THEORY}

The so-called PDLC film is a mixture of LC and monomers in an appropriate proportion. The monomers are induced by external light or heat and have a polymerization capability to form polymers. However, the solubility of the LC molecules decreases with the degree of polymerization. When the solubility is less than a certain value, phase separation will occur, and the LC molecules are precipitated. Finally, the LC molecules are dispersed amongst the polymer. This phase separation method is called as PIPS [14-17].

The main purpose of the procedure is to reduce the solubility of the LC molecules and generate phase separation by consuming monomer molecules by polymerization. The polymerization process transforms the monomer into the whole polymer network is as follows. Addition polymerization is the addition of monomer molecules to the growing chain, and no small molecules are precipitated during the reaction. The main characteristic of step polymerization is the formation of two dimers by matching monomer interactions. The polymer chain then is formed gradually from the two dimers or unreactive monomers.

The polymerization used in PDLC films is mostly additive polymerization and step polymerization. In the early stage of addition polymerization, there are usually three ways to initiate polymerization, cationic, anionic, and free radical reactions. Free radical reaction is the most widely used method, which can make one end of the double bond of the monomer molecule chemically active by external inducement. Then, it can bond with other molecules. One end of the bonded molecule is still chemically active, and it will interact with other monomers. This process will be repeated continuously until the reaction terminates [14-17].

The dynamic process of addition polymerization can be divided into initiation, propagation and termination stages. In the initial process, the initiator decomposes free radicals by external light or heat excitation. However, it is easily attacked by free radicals because of the low stability of the carbon-hydrogen double bonds at both ends of the monomer molecule. This will lead to free radicals being attached to the attacked monomers and will make the other end of the monomer produce free radicals. In the process of propagation, the free radicals of the attacked monomer will attack another monomer and repeat the reaction to achieve rapid link polymerization until the monomers have been depleted or free radicals interact with each other, thereby reducing their own activity and ultimately reaching the end of the reaction. When the PDLC film is formed, the monomer is rapidly polymerized and phase separation also happens.

The operation principle of PDLCs for light modulation is as shown in Fig. 2. In the voltage-off condition, as shown in Fig. 2 a, the LC droplets are randomly oriented. 
The effective refractive index $n_{\text {eff }}$ seen by the light will be different to that of the polymer networks $n_{p}$. Hence, the incident light is scattered mainly in the forward direction, or it is reflected. In the voltage-on state, the LC molecules are reoriented along the electric field direction, as shown in Fig. 2 b. Therefore, the ordinary refractive index of the LC, $n_{o}$, is the refractive index seen by the light, and $n_{p}$ usually is very close to $n_{o}$ do light passes through the PDLC layer with high transmission and less scattering.

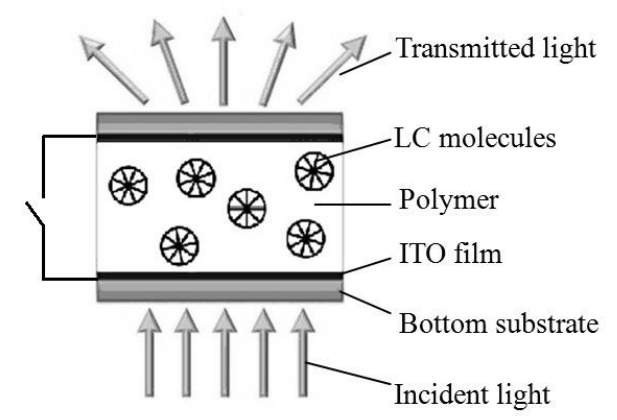

a

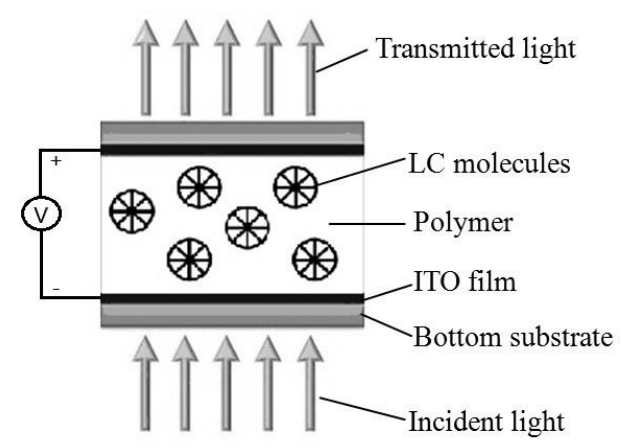

b

Fig. 2. Operation mechanism of PDLC for light modulation, where the director of LC is radial type: a-in the state of power off; $b$-in the state of power on, and the direction of the applied voltage is as shown

\section{RESULTS AND DISCUSSION}

The system of measurement, EOT-01, was developed by the Changchun Institute of Optics, Fine Mechanics and Physics, Chinese Academy of Sciences. Following the steps of the approach, a halogen lamp was used as a light source for characterizing the optical transmission properties of the new diffuser. A function generator provided an AC electric field (square wave, frequency of $1 \mathrm{kHz}$, voltage of $0-10 \mathrm{Vrms}$ ) across the cell thickness. The threshold voltage and the driving voltage are defined as the voltages at which the transmitted light power is increased to $10 \%$ and $90 \%$ of the initial value at a null voltage, respectively. In Fig. 3, the curve of the optical transmittance-time for PDLC cells is shown. There are some auxiliary lines to present the measured data of the PDLC cells. The range of $\tau_{\text {on }}$ was about $10.5 \mathrm{~ms}$. On the other hand, the $\tau_{\text {off }}$ was about $10.5 \mathrm{~ms}$. Those data can be calculated directly from Fig. 3. The response time $\left(\tau_{\text {on }}+\tau_{\text {off }}\right)$ was about $21.0 \mathrm{~ms}$. Larger LC droplets can be acquired under the described fabrication procedure.
Therefore, the response time of the LC device is improved significantly, compared to conventional types. The reasons are that the thickness of the PDLC film is optimized and the fabrication process is conducive for the production of larger LC droplets.

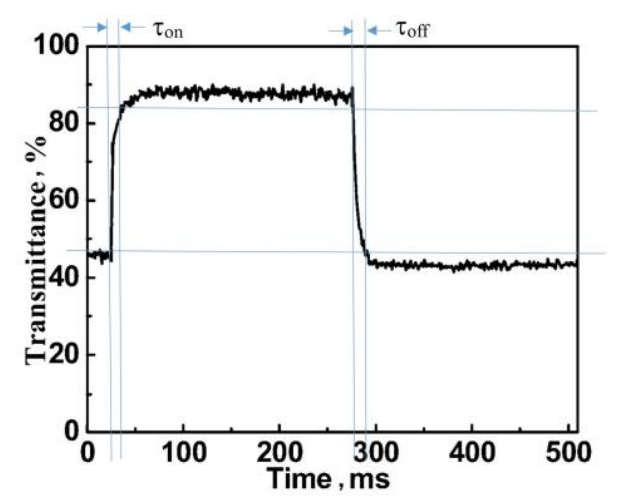

Fig. 3. Response times of the new diffuser based on PDLC film under $10 \mathrm{Vrms}$ and $1 \mathrm{kHz}$

Under the condition of $10 \mathrm{Vrms}$ and $1 \mathrm{kHz}$, the transmittance of the new diffuser based on the PDLC film was as shown in Fig. 4. The system of measurement, EOT-01, was still utilized to measure the classic electrooptical characteristics of the fabricated LC cells. From those data, the proposed diffuser was observed to exhibit good transmission in the visible wavelength range. When the operation voltage was greater than the threshold value, the transmittance of the PDLC film was greater than $60 \%$. That means that, in general, the PDLC film had a good transmissive imaging response.

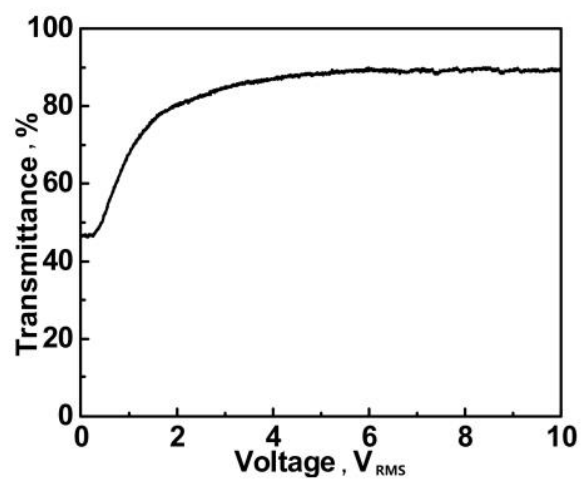

Fig. 4. Transmittance of the proposed diffuser based on PDLC film under $10 \mathrm{Vrms}$ and $1 \mathrm{kHz}$

Another testing arrangement to evaluate the transmissive imaging features of the new diffuser was set up, as shown in Fig. 5. The diffuser based on PDLC film was between the background and a CCD (Charge Coupled Device). Light was passed through the PDLC cell and fell on the CCD. The CCD was 3 Megapixel in resolution, $1 / 2$ inch in thickness, and $6.4 \mathrm{~mm} \times 4.8 \mathrm{~mm}$ in area, and was connected to a PC. A tuneable externally-applied voltage (0 Vrms - $10 \mathrm{Vrms})$ at $1 \mathrm{kHz}$ was used to drive the PDLC cell. In order to get the corresponding polarization information for the new diffuser, a polarizer was set in front of the CCD.

Using the experimental set-up shown in Fig. 5, 2D images of a scene can be captured and the polarization 
state of those images, modulated by the polarizer, can be obtained, as shown in Fig. 6.
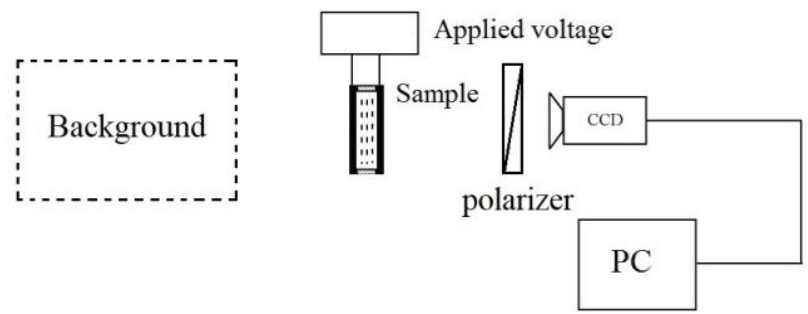

Fig. 5. Transmissive imaging test arrangement

It can be observed that if the background was subjected to an s-polarization wave, serious atomization will occur. Many objects in the scene could not be seen. Under this condition, the transmissive imaging was not very good. If the background was subjected to a ppolarization wave, the transmissive imaging was much clearer. When observing objects in natural light, the result was superimposed by the two kinds of polarization waves. For those 2D images in Fig. 6, many objects in the rear scene could be observed. Further observation showed that the contrast of the 2D image decreased significantly under natural light conditions. This means that a polarizer was needed to increase contrast and much more information was obtained to make imaging much clearer.

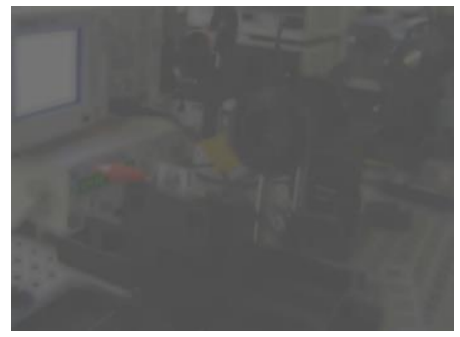

a

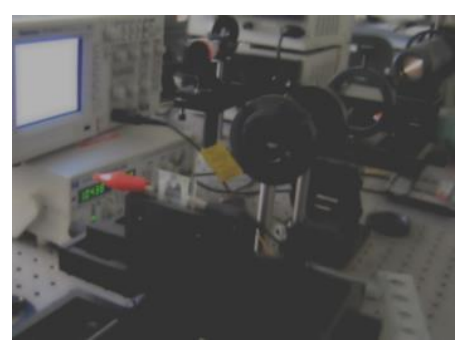

b

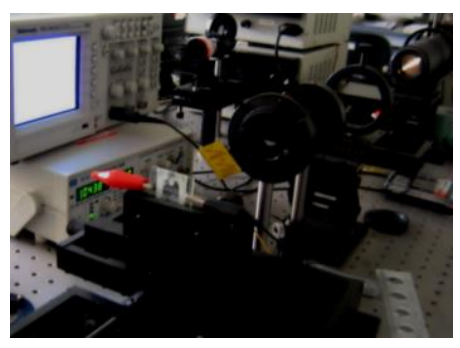

$\mathrm{c}$

Fig. 6. Results of transmissive imaging under different polarization states: a-the result for an s-polarization wave; $b$-the result for a p-polarization wave; $c$-the result for natural light

The purpose of testing s-polarization wave and p-polarization waves was to obtain much more information for future applications about the new diffuser in different environments.

On the basis of the test setup shown in Fig. 5, a $532 \mathrm{~nm}$ laser, a patterned template, an imaging lens, and a half-wave plate were added in front of the sample. In this way, a test to obtain the see-through projection effect of the designed diffuser was set up. During the experiment, a CCD was utilized to obtain $2 \mathrm{D}$ images at different angles along the central axis of the sample.

The incident light was projected onto the sample through the imaging system. The projection effect was taken as shown in Fig. 7. The patterned template was chosen as a square with a circular point. The circular point was present in the middle of the square. Those patterns had been hollowed out of the template. Those projection images were taken in two different kinds of polarization states. The 2D images at different polarization states were taken from the angles of $0,10,20$ and 30 degrees, respectively. From the obtained results, it can be observed that much better quality and brightness images could be obtained under the condition of the s-polarization wave as the light source. Under this condition, the brightness of the image will be relatively reduced when the brightness is greater. The small angle can obtain better image intensity, and the small angle of view is much more suitable for an optical diffuser. Therefore, only one polarized light of PDLC film without applied voltage will be scattered. When the applied electric field is loaded, the PDLC film will become clear and transparent, and it has the polarization-dependence feature.

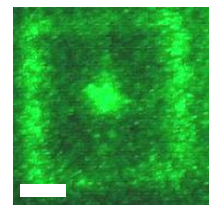

a

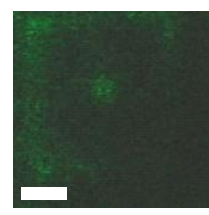

e

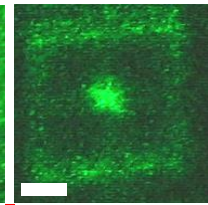

b

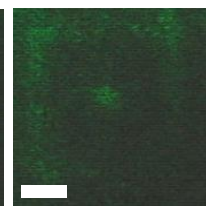

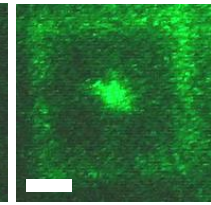

c

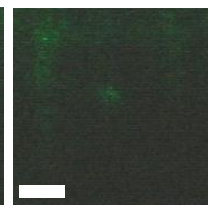

g

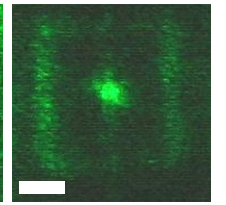

d

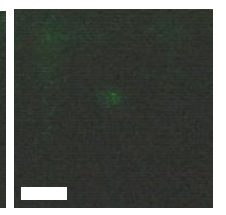

$\mathrm{h}$
Fig. 7. Imaging results for the see-through projection effect of the new PDLC diffuser under different conditions: $a-d$ are under s-polarization wave, the angle of the observation was $0^{\circ}, 10^{\circ}, 20^{\circ}$, and $30^{\circ}$, respectively; $\mathrm{e}-\mathrm{h}$ are under $\mathrm{p}$ polarization wave, the angle of the observation was $0^{\circ}, 10^{\circ}, 20^{\circ}$, and $30^{\circ}$, respectively. The size of the scale bar is $1 \mathrm{~cm}$

\section{CONCLUSIONS}

In the present investigation, the electro-optical characteristics of a new type of diffuser prepared by PDLC were described in detail. An LC cell was made from PDLC that can be used as a diffuser without the application of an electric field and can become a transparent screen after applying an electric field. Background scenery cannot be observed when the diffuser is operating in the electric field-off condition, but the scenery can be clearly observed in the polarized state in the absence of scattering. 
However, for natural light, the degree of contrast was lower. That was because the new PDLC diffuser can be adjusted to obtain a specific effect. Thus, the new-type diffuser can play a role in many applications. In projection tests, it was demonstrated that the image brightness of the diffuser was higher at small angles and was much lower at large angles. It is envisaged that the new diffuser could be used in some augmented reality and mixed reality applications. However, this was a preliminary study and future work will build on these initial findings.

\section{Acknowledgments}

This work was supported by a grant from the Natural Science Foundation of China (51703071, 61771353), Natural Science Foundation of Hubei Province (2019CFB553), the Research Foundation of Education Bureau of Hubei Province China (D20171504), and the Open Foundation for Hubei Key Laboratory of Intelligent Robot (HBIR 201805, 201901).

\section{REFERENCES}

1. Gao, H.Y., Yao, Q.X., Liu, P., Zheng, Z.Q., Liu, J.C., Zheng, H.D., Zeng, C., Yu, Y.J., Sun, T., Zeng, Z.X. Latest Development of Display Technologies Chinese Physics B 25 (9) 2016: pp. 1-8. https://10.1088/1674-1056/25/9/094203

2. Kim, S.K., Kim, D.W., Kwon, Y.M., Son, J.Y. Evaluation of the Monocular Depth Cue in 3D Displays Optics Express 16 (26) 2018: pp. 21415-21422. https://10.1364/oe.16.021415

3. Lv, G.J., Wang, J., Zhao, W.X., Wang, Q.H. ThreeDimensional Display Based on Dual Parallax Barriers with Uniform Resolution Applied Optics 52 (24) 2013: pp. $6011-6015$. https://doi.org/10.1364/AO.52.006011

4. Carmigniani, J., Furht, B., Anisetti, M., Ceravolo, P., Damiani, E., Ivkovic, M. Augmented Reality Technologies, Systems and Applications Multimedia Tools and Applications 51 (1) 2011: pp. 341-377. https://10.1007/s11042-010-0660-6

5. Tepper, O.M., Rudy, H.L., Lefkowitz, A., Weimer, K.A., Marks, S.M., Stern, C.S., Garfein, E.S. Mixed Reality with HoloLens: Where Virtual Reality Meets Augmented Reality in the Operating Room Plastic and Reconstructive Surgey 140 (5) 2017: pp. 1066-1072. https://10.1097/prs.0000000000003802

6. Sekiguchi, Y., Konno, A., Tsumura, M. Design Method for a Thin Uniform Direct Backlight Using a Diffuser Plate with a Pattern on the Surface and Optical Films Applied Optics 54 (3) 2015: pp. $482-491$. https://10.1364/ao.54.000482
7. Lehtolahti, J., Kuittinen, M., Turunen, J., Tervo, J. Coherence Modulation by Deterministic Rotating Diffusers Optics Express 23 (8) 2015: pp. 10453-10466. https://10.1364/oe.23.010453

8. Geng, J. Three-Dimensional Display Technologies Advances in Optics and Photonics 5(4) 2013: pp. $456-535$. https://10.1364/aop.5.000456

9. Chellappan, K.V., Erden, E., Urey, H. Laser-Based Displays: A Review Applied Optics 49 (25) 2010: pp. F79-F98. https://10.1364/ao.49.000f79

10. Leith, E.N., Upatnieks, J. Holographic Imagery Through Diffusing Media Journal of the Optical Society of America 56 (4) 1966: pp. $523-523$. https://10.1364/josa.56.000523

11. Huang, T.C., Ciou, J.R., Huang, P.H., Hsieh, K.H., Yang, S.Y. Fast Fabrication of Integrated Surface-Relief and Particle-Diffusing Plastic Diffuser by Use of a Hybrid Extrusion Roller Embossing Process Optics Express 16 (1) 2008: pp. 440-447. https://10.1364/oe.16.000440

12. Manninen, P., Koskela, T., Ylianttila, L., Kärhä, P., Ikonen, E. Estimation of the Optical Receiving Plane Positions of Solar Spectroradiometers with Spherical Diffusers on the Basis of Spatial Responsivity Data Optics Letters 34 (20) 2009: pp. 3241-3243. https://10.1364/ol.34.003241

13. Schmid, T., Hornung, T., Nitz, P. Calibration Function for an Experimental Setup for the Measurement of Irradiance Distributions via a Transmitting Diffuser Applied Optics 54 (4) 2015: pp. $927-933$. https://10.1364/ao.54.000927

14. Cupelli, D., Nicoletta, F.P., Manfredi, S., Vivacqua, M., Formoso, P., Filpo, G.D., Chidichimo, G. Self-Adjusting Smart Windows Based on Polymer-Dispersed Liquid Crystals Solar Energy Materials and Solar Cells 93 (11) 2009: pp. $2008-2012$. https://10.1016/j.solmat.2009.08.002

15. Liu, Y.J., Ding, X.Y., Lin, S.C.S., Shi, J.J., Chiang, I.K., Huang, T. J. Surface Acoustic Wave Driven Light Shutters Using Polymer-Dispersed Liquid Crystals Advanced Materials 23 (14) 2011: pp. 1656-1659. https://10.1002/adma.201003708

16. Li, H., Pan, F., Wu, Y.T., Zhang, Y.D., Xie, X.L. Polarization-Independent Electrically Tunable Holographic Polymer Dispersed Liquid Crystals Grating Doped with Chiral Molecules Materials Science (Medžiagotyra) 23 (2) 2017: pp. $114-118$. https://10.5755/j01.ms.23.2.16312

(c) Li et al. 2021 Open Access This article is distributed under the terms of the Creative Commons Attribution 4.0 International License (http://creativecommons.org/licenses/by/4.0/), which permits unrestricted use, distribution, and reproduction in any medium, provided you give appropriate credit to the original author(s) and the source, provide a link to the Creative Commons license, and indicate if changes were made. 